\title{
Scale and watershed features determine lake chemistry patterns across physiographic regions in the far north of Ontario, Canada
}

\author{
Josef MACLEOD, ${ }^{1}$ Wendel (Bill) KELLER, ${ }^{1 *}$ Andrew M. PATERSON, ${ }^{2}$ Richard D. DYER, ${ }^{3}$ John M. GUNN ${ }^{1}$
}

${ }^{1}$ Cooperative Freshwater Ecology Unit, Vale Living with Lakes Centre, Laurentian University, 935 Ramsey Lake Road, Sudbury, Ontario, P3E 2C6; ${ }^{2}$ Ontario Ministry of the Environment and Climate Change, Dorset Environmental Science Centre, 1026 Bellwood Acres Road, Dorset, Ontario P0A 1E0; ${ }^{3}$ Earth Resources and Geoscience Mapping Section, Ontario Geological Survey, 933 Ramsey Lake Road, Sudbury, Ontario, P3E 6B5

*Corresponding author: bkeller@laurentian.ca

ABSTRACT

Changes in the far north of Ontario $\left(>50^{\circ} \mathrm{N}\right.$ latitude), like climate warming and increased industrial development, will have direct effects on watershed characteristics and lakes. To better understand the nature of remote northern lakes that span the Canadian Shield and Hudson Bay Lowlands, and to address the pressing need for limnological data for this vast, little-studied area of Ontario, lake chemistry surveys were conducted during 2011-2012. Lakes at the transition between these physiographic regions displayed highly variable water chemistry, reflecting the peatland landscape with a mix of bog and fen watersheds, and variations in the extent of permafrost. In the transition area, Shield and Lowlands lakes could not be clearly differentiated based on water chemistry; peat cover decouples, to varying degrees, the lakes from the influences of bedrock and surficial deposits. Regional chemistry differences were apparent across a much broader area of northern Ontario, due to large-scale spatial changes in geology and in the extent of peatlands and permafrost. Shield lakes in the far northwest of Ontario had Ca, Mg, and TP concentrations markedly higher than those of many Lowlands lakes and previously studied Shield lakes south of $50^{\circ} \mathrm{N}$, related to an abundance of lacustrine and glacial end-moraine deposits in the north.

Key words: Lake; chemistry; Canadian Shield; Hudson Bay Lowlands; Ring of Fire.

Received: July 2016. Accepted: October 2016.

\section{INTRODUCTION}

The far north of Ontario $\left(>50^{\circ} \mathrm{N}\right)$, straddles two physiographic regions, the Hudson Bay Lowlands (underlain by Paleozoic age bedrock) and the Canadian Shield (underlain by Archean age bedrock). Although very different in geological setting and vegetation cover, both regions contain vast numbers of freshwater lakes which are a vital component of the health of northern environments and which provide sustenance and essential services to northern peoples. A recent estimate by the Ontario Ministry of Natural Resources and Forestry (MNRF) identified $\sim 708,000$ lakes in the far north of Ontario with 118,000 on the Shield and 590,000 on the Lowlands (MNRF 2015, personal communication).

Lakes in northern Ontario are very vulnerable to future climate change (Far North Science Advisory Panel, 2010). Climate warming will be most pronounced in northern areas of the province (Colombo et al., 2007), and recent paleolimnological studies of diatoms (Rühland et al., 2013, 2014) and cladocerans (Jeziorski et al., 2015) indicate that warming-related community changes are already happening in northern Ontario lakes. As well, future largescale mining activity and associated infrastructure devel- opment is inevitable for northern Ontario, with the discovery of massive metal deposits in the 'Ring of Fire' (ROF) area (Hjartarson et al., 2014).

Given the increasing interest in development throughout the north, and in the ROF area in particular, there is a need to improve our basic understanding of northern aquatic ecosystems so that we may understand how future impacts may affect them. Conserving the diversity, function, and provision of aquatic ecosystem services in northern Ontario in the face of climate change and industrial development requires sound scientific data from which to make informed management decisions. Currently, few data exist for the remote lakes in the far north of Ontario, one of the least disturbed and largest $\left(\sim 452,000 \mathrm{~km}^{2}\right)$ ecosystems on earth. Only two studies have documented lake chemistry in this area during recent decades, in a limited number of lakes (Keller and Pitblado, 1989; Paterson et al., 2014). An earlier chemistry survey of Ontario lakes did include some lakes north of $50^{\circ} \mathrm{N}$, but considered only alkalinity and total dissolved solids (Ryder, 1964). Due to a lack of available data, subarctic lakes in Ontario were conspicuously absent from an extensive review of the limnology of Arctic and Subarctic lakes and ponds across the circumpolar area (Rautio et al., 2011). We seek to help fill this im- 
portant knowledge gap for northern aquatic ecosystems.

The boundary between the Canadian Shield and the Hudson Bay Lowlands (Royal Commission on the Northern Environment, 1985; Far North Science Advisory Panel, 2010; Riley, 2011), is coincident with the unconformable contact between older Precambrian basement rock and younger (overlying) Paleozoic age sedimentary rock. The region is covered by a thick mantle of peat, and Quaternary glacial and marine deposits (up to $76 \mathrm{~m}$ thick), creating a complex surficial landscape (Dyer and Handley, 2013) and making the precise delineation of the boundary difficult. However, at a coarse scale this operational boundary is useful for general comparisons between physiographic regions. The ROF, a geological formation consisting of both volcanic and intrusive igneous rock (Metsaranta and Houle, 2012) is located at the Shield/Lowlands boundary. The $\mathrm{ROF}$ is an area rich in mineral deposits including nickel, copper, zinc and chromite. These Ni-Cu-platinum group element deposits were first discovered by a mineral exploration program in 2007 . Since the discovery, mineral claims have greatly expanded in this region and assessments for possible mines are ongoing.

Surveys were conducted in 2011 and 2012 to collect water chemistry data on Shield and Lowlands lakes in the ROF area and extending more broadly across northern Ontario. The objectives of the study were to establish the ranges of chemical variability of lakes in the ROF area and identify factors affecting this variability; and to determine if water chemistry differentiates Shield and Lowlands lakes within the ROF area, or across northern Ontario above $50^{\circ} \mathrm{N}$.

\section{METHODS}

\section{ROF survey}

Between August 13 and 15, 2011, single point water samples from 98 lakes (21 on the Shield, 77 on the Lowlands) in the ROF area (Fig. 1) were collected by the Ontario Geological Survey (OGS) following methods from Dyer (2011). The survey area was located 40-140 km east of the community of Webequie. Shield lakes were all within $10 \mathrm{~km}$ of the Shield/Lowlands boundary.

A helicopter on floats was used to travel to the lakes. Water samples for laboratory analyses were collected from a depth of 0.5-1.0 m by a weighted intake hose connected to a diaphragm pump inside the helicopter. Some water quality parameters, including $\mathrm{pH}$ and conductivity, were measured at each lake using a flow cell attached to a YSI model 600xl multi-parameter probe. Samples for laboratory analyses were kept in coolers. Total phosphorus (TP), three measures of nitrogen (total Kjeldahl nitrogen, combined ammonia and ammonium, combined nitrate and nitrite), dissolved organic carbon (DOC) con- centrations and true colour were analysed at the Ontario Ministry of the Environment and Climate Change (MOECC) laboratory in Dorset, Ontario using standard methods (OMOE, 1983). Metals and major ions were analysed by the OGS geosciences laboratory in Sudbury, Ontario (OGS, 2007).

\section{Large scale survey}

During July 11-15, 2012, a second survey was performed by Laurentian and Queen's universities of a smaller number of lakes $(\mathrm{N}=29 ; 14$ on the Shield and 15 on the Lowlands) across a much broader section of northern Ontario (Fig. 1). Lakes were sampled at a central location from a fixed-wing aircraft on amphibious floats. Lake depth was determined using a sonar depth sounder. A water sample was obtained at each lake using a composite depth sampling device consisting of a large ( $\sim \mathrm{L}$ ) plastic bottle with a restricted inlet that allowed water to enter at a slow rate. The sampler was rinsed with lake water, then lowered to the Secchi depth or $1 \mathrm{~m}$ off bottom (which ever was shallower) and slowly retrieved, allowing the bottle to fill evenly across all depths. The sample water was filtered through an $80 \mu \mathrm{m}$ mesh Nalgene ${ }^{\circledR}$ funnel into a $6 \mathrm{~L}$ plastic sample container. These composite water samples were kept in coolers and later subsampled for subsequent laboratory analyses at the Dorset MOECC Laboratory (OMOE, 1983).

In 2012 (May 14-August 25), the MNRF Broadscale Monitoring Program (BSM) sampled 20 lakes (16 Shield and 4 Lowlands) in northern Ontario which were added to the data set (Fig. 1). Comparable collection methods to the Laurentian/Queen's 2012 survey were employed (Sandstrom et al., 2011) and samples were also analysed at the Dorset MOECC laboratory (OMOE, 1983).

\section{Data screening, combining, and analyses}

The 2012 data from the Laurentian/Queen's survey were pooled with data from the 2012 BSM survey by MNRF to form the Large Scale Survey data set, which was analysed separately from the data for the ROF survey. Variables analysed for the Large Scale Survey were the same as examined for the 2011 ROF survey as well as dissolved inorganic carbon (DIC).

Spearman's correlation coefficients were used to identify the basic patterns in the data and to identify where variables described overlapping variance (co-variates). Typically, a sequential Bonferroni adjustment is used with multiple comparisons. However, this method has a number of problems when applied to ecological data (Moran, 2003). We used an $\alpha$ criterion of 0.01 to account for increased error from multiple correlations, without being so conservative that most correlations would be eliminated from significance.

Principle Components Analysis (PCA) on data from 
the ROF and Large Scale Surveys was used to investigate dominant patterns using an eigenvector based ordinal approach. Variables were $\log _{10}$ transformed when necessary to achieve the best fit to normal distributions. PCA has long been effectively used in environmental studies (Keller and Pitblado, 1989; Keller and Conlon, 1994; Medeiros et al., 2012). By plotting the individual lake scores for the first two principle components, a two-dimensional representation of each lake's characteristics was obtained. The lakes were plotted by physiographic region to illustrate the general relationships to the landscape.

We used non-parametric permutation tests to examine spatial variation among the lakes (Clarke, 1993; Anderson, 2001). Analysis of Similarities (ANOSIM) was used to test for overall differences between Shield and Lowlands lakes (Oliver and Beattie, 1996; Chapman and Underwood, 1999). Mann-Whitney U-tests were used to identify where differences existed for individual variables between the Shield and Lowlands lake groups.

\section{RESULTS}

\section{ROF survey}

The lakes in this survey were all shallow ( $\leq 5 \mathrm{~m}$ maximum depth); however, they showed highly diverse water chemistry characteristics. Conductivity $\left(7-161 \mu \mathrm{S} \mathrm{cm}^{-1}\right)$, colour (13.6-195.0 TC units), inorganic $\mathrm{N}\left(6-156 \mu \mathrm{g} \mathrm{L}^{-1}\right)$, Si $\left(0.02-2.36 \mathrm{mg} \mathrm{L}^{-1}\right), \mathrm{Ca}\left(0.48-28.07 \mathrm{mg} \mathrm{L}^{-1}\right), \mathrm{Fe}(0.01-$ $\left.1.13 \mathrm{mg} \mathrm{L}^{-1}\right)$ and $\mathrm{Mg}\left(0.19-5.05 \mathrm{mg} \mathrm{L}^{-1}\right)$ all showed more than an order of magnitude difference between maximum and minimum values (Tab. 1; Supplementary Tab. 1).

Chemistry and morphometry variables revealed some strong $(\mathrm{P}<0.01)$ associations. Lake area was positively correlated with $\mathrm{pH}, \mathrm{TP}$, conductivity and $\mathrm{Ca}$ and negatively correlated with DOC and colour. Lake depth was negatively correlated with DOC, colour, total N, inorganic N, Si and Mg (Supplementary Tab. 2a). PCA illustrated that the Shield and Lowlands lake groups overlapped to a con-

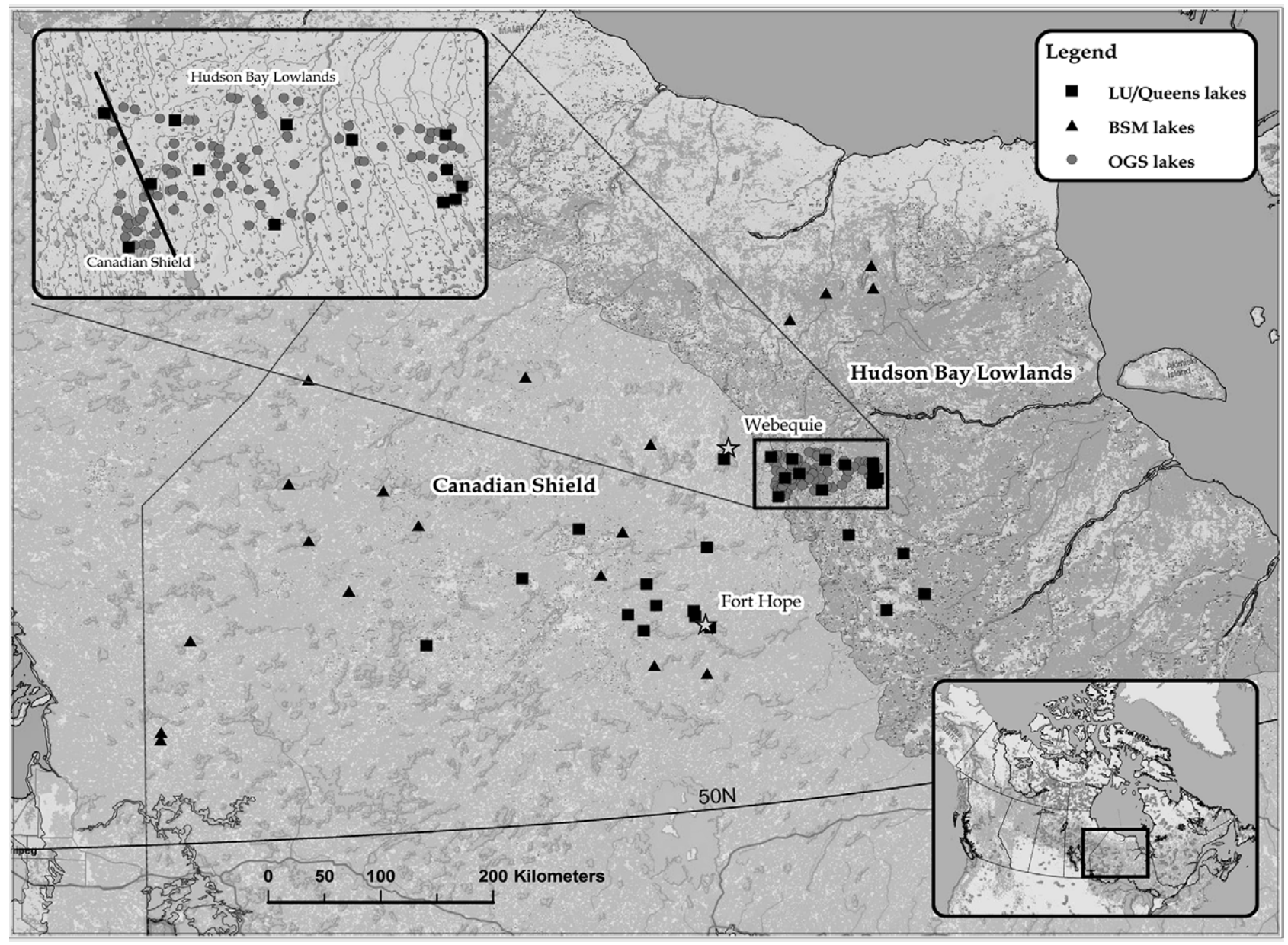

Fig. 1. Map of the study areas showing the locations of the ROF lakes sampled in 2011 by OGS (OGS lakes; see inset), and the 2012 Large Scale Survey lakes sampled by Laurentian and Queen's universities (LU/ Queen's lakes) and MNRF (BSM lakes). 
siderable degree, with Shield lakes displaying a clustered, less variable group within a more diverse group of Lowlands lakes (Fig. 2). The lakes oriented along axes which generally corresponded with $\mathrm{pH}$-major ions and colourDOC, respectively. The variables which scored most positively on PC1 (36\% of variation explained) were $\mathrm{Mg}$ (0.426), $\mathrm{Ca}(0.420)$, conductivity (0.417) and $\mathrm{pH}(0.393)$; while PC2 (19.5\% of variation explained) had high positive scores for colour (0.537), DOC (0.523) and Fe (0.486). Total P scored negatively on PC2 (-0.167). ANOSIM showed no overall difference (global $\mathrm{R}=-0.068, \mathrm{P}=0.845$ ) between Shield and Lowlands lakes. The negative value of the observed $\mathrm{R}$ for the ROF lakes indicates that there is a greater similarity between the two groups than there is within each group (Chapman and Underwood, 1999). This reflects the fact that the Lowlands group had very high

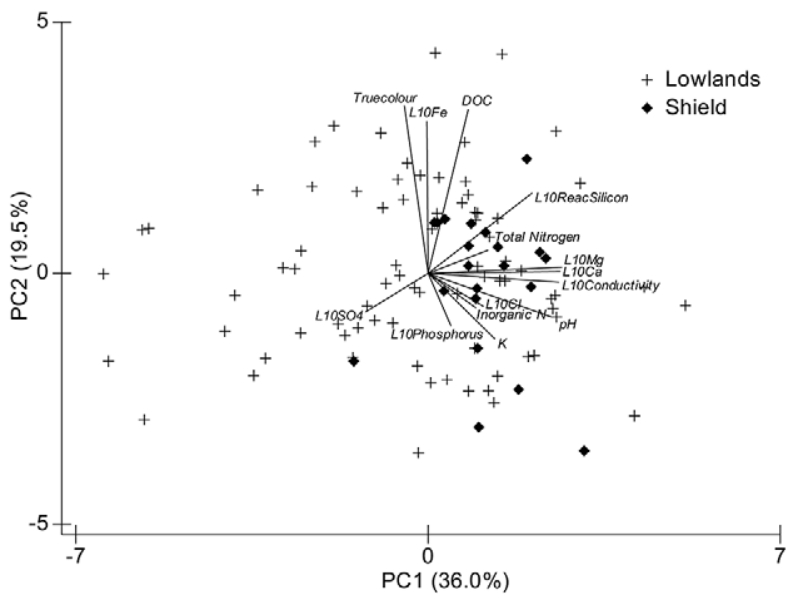

Fig. 2. PCA of 2011 water chemistry and morphometry for the ROF lakes ( $\mathrm{N}=98,21$ Shield and 77 Lowlands).

Tab. 1. Descriptive statistics for lake chemistry and morphometry for the 2011 ROF Survey (N=98, 77 Lowlands and 21 Shield).

\begin{tabular}{|c|c|c|c|c|c|c|c|c|}
\hline Variable & $\begin{array}{c}\text { Mann- } \\
\text { Whitney } \\
\text { U-test (P) }\end{array}$ & Region & Mean & Median & Max & Min & $\begin{array}{l}\text { Standard } \\
\text { deviation }\end{array}$ & $\begin{array}{l}\text { Coefficient } \\
\text { of variation }\end{array}$ \\
\hline Lake depth (m) & 0.029 & $\begin{array}{c}\text { Lowland } \\
\text { Shield }\end{array}$ & $\begin{array}{l}1.82 \\
1.39\end{array}$ & $\begin{array}{l}1.50 \\
1.30\end{array}$ & $\begin{array}{l}5.00 \\
2.50\end{array}$ & $\begin{array}{l}0.50 \\
0.90\end{array}$ & $\begin{array}{l}0.87 \\
0.42\end{array}$ & $\begin{array}{l}0.48 \\
0.30\end{array}$ \\
\hline Lake area (ha) & 0.880 & $\begin{array}{l}\text { Lowland } \\
\text { Shield }\end{array}$ & $\begin{array}{l}95.01 \\
90.89\end{array}$ & $\begin{array}{l}32.00 \\
29.84\end{array}$ & $\begin{array}{c}1081.71 \\
738.16\end{array}$ & $\begin{array}{l}5.09 \\
9.31\end{array}$ & $\begin{array}{l}184.08 \\
167.96\end{array}$ & $\begin{array}{l}1.94 \\
1.85\end{array}$ \\
\hline Conductivity $\left(\mu \mathrm{S} \mathrm{cm}^{-1}\right)$ & 0.060 & $\begin{array}{l}\text { Lowland } \\
\text { Shield }\end{array}$ & $\begin{array}{l}41.55 \\
45.95\end{array}$ & $\begin{array}{l}34.00 \\
45.00\end{array}$ & $\begin{array}{c}161.00 \\
79.00\end{array}$ & $\begin{array}{c}7.00 \\
19.00\end{array}$ & $\begin{array}{l}31.05 \\
15.59\end{array}$ & $\begin{array}{l}0.75 \\
0.34\end{array}$ \\
\hline $\mathrm{pH}$ & 0.004 & $\begin{array}{l}\text { Lowland } \\
\text { Shield }\end{array}$ & $\begin{array}{l}7.26 \\
7.44\end{array}$ & $\begin{array}{l}6.97 \\
7.26\end{array}$ & $\begin{array}{l}8.28 \\
8.10\end{array}$ & $\begin{array}{l}4.24 \\
6.75\end{array}$ & - & - \\
\hline $\mathrm{DOC}\left(\mathrm{mg} \mathrm{L}^{-1}\right)$ & 0.143 & $\begin{array}{l}\text { Lowland } \\
\text { Shield }\end{array}$ & $\begin{array}{l}14.63 \\
15.54\end{array}$ & $\begin{array}{l}14.10 \\
15.60\end{array}$ & $\begin{array}{l}24.40 \\
19.00\end{array}$ & $\begin{array}{c}5.60 \\
12.00\end{array}$ & $\begin{array}{l}3.69 \\
1.91\end{array}$ & $\begin{array}{l}0.25 \\
0.12\end{array}$ \\
\hline True colour (TCU) & 0.140 & $\begin{array}{l}\text { Lowland } \\
\text { Shield }\end{array}$ & $\begin{array}{l}81.63 \\
67.87\end{array}$ & $\begin{array}{l}79.80 \\
72.60\end{array}$ & $\begin{array}{l}195.00 \\
111.00\end{array}$ & $\begin{array}{l}13.60 \\
15.80\end{array}$ & $\begin{array}{l}37.43 \\
23.55\end{array}$ & $\begin{array}{l}0.46 \\
0.35\end{array}$ \\
\hline Inorganic $\mathrm{N}\left(\mu \mathrm{g} \mathrm{L}^{-1}\right)$ & 0.496 & $\begin{array}{l}\text { Lowland } \\
\text { Shield }\end{array}$ & $\begin{array}{l}46.03 \\
48.00\end{array}$ & $\begin{array}{l}40.00 \\
48.00\end{array}$ & $\begin{array}{c}156.00 \\
80.00\end{array}$ & $\begin{array}{c}6.00 \\
26.00\end{array}$ & $\begin{array}{l}20.68 \\
15.86\end{array}$ & $\begin{array}{l}0.45 \\
0.33\end{array}$ \\
\hline Total N $\left(\mu \mathrm{g} \mathrm{L}^{-1}\right)$ & 0.000 & $\begin{array}{l}\text { Lowland } \\
\text { Shield }\end{array}$ & $\begin{array}{l}447.56 \\
526.29\end{array}$ & $\begin{array}{l}434.00 \\
504.00\end{array}$ & $\begin{array}{l}703.00 \\
711.00\end{array}$ & $\begin{array}{l}254.00 \\
417.00\end{array}$ & $\begin{array}{l}95.62 \\
75.76\end{array}$ & $\begin{array}{l}0.21 \\
0.14\end{array}$ \\
\hline Total $\mathrm{P}\left(\mu \mathrm{g} \mathrm{L} \mathrm{L}^{-1}\right)$ & 0.109 & $\begin{array}{l}\text { Lowland } \\
\text { Shield }\end{array}$ & $\begin{array}{l}17.02 \\
14.13\end{array}$ & $\begin{array}{l}14.30 \\
12.30\end{array}$ & $\begin{array}{l}44.40 \\
27.90\end{array}$ & $\begin{array}{l}7.20 \\
4.80\end{array}$ & $\begin{array}{l}7.58 \\
5.69\end{array}$ & $\begin{array}{l}0.44 \\
0.40\end{array}$ \\
\hline Reactive Si (mg L $\left.{ }^{-1}\right)$ & 0.004 & $\begin{array}{l}\text { Lowland } \\
\text { Shield }\end{array}$ & $\begin{array}{l}0.58 \\
0.95\end{array}$ & $\begin{array}{l}0.44 \\
0.98\end{array}$ & $\begin{array}{l}2.36 \\
2.00\end{array}$ & $\begin{array}{l}0.02 \\
0.12\end{array}$ & $\begin{array}{l}0.56 \\
0.56\end{array}$ & $\begin{array}{l}0.95 \\
0.59\end{array}$ \\
\hline $\mathrm{Ca}\left(\mathrm{mg} \mathrm{L}^{-1}\right)$ & 0.099 & $\begin{array}{l}\text { Lowland } \\
\text { Shield }\end{array}$ & $\begin{array}{l}7.60 \\
8.32\end{array}$ & $\begin{array}{l}6.47 \\
8.56\end{array}$ & $\begin{array}{l}28.07 \\
14.16\end{array}$ & $\begin{array}{l}0.48 \\
3.00\end{array}$ & $\begin{array}{l}5.78 \\
2.89\end{array}$ & $\begin{array}{l}0.76 \\
0.35\end{array}$ \\
\hline $\mathrm{Cl}\left(\mathrm{mg} \mathrm{L}^{-1}\right)$ & 0.758 & $\begin{array}{l}\text { Lowland } \\
\text { Shield }\end{array}$ & $\begin{array}{l}0.19 \\
0.16\end{array}$ & $\begin{array}{l}0.15 \\
0.15\end{array}$ & $\begin{array}{l}1.25 \\
0.26\end{array}$ & $\begin{array}{l}0.02 \\
0.05\end{array}$ & $\begin{array}{l}0.17 \\
0.05\end{array}$ & $\begin{array}{l}0.86 \\
0.34\end{array}$ \\
\hline $\mathrm{Fe}\left(\mathrm{mg} \mathrm{L}^{-1}\right)$ & 0.049 & $\begin{array}{l}\text { Lowland } \\
\text { Shield }\end{array}$ & $\begin{array}{l}0.10 \\
0.06\end{array}$ & $\begin{array}{l}0.07 \\
0.05\end{array}$ & $\begin{array}{l}1.13 \\
0.14\end{array}$ & $\begin{array}{l}0.01 \\
0.01\end{array}$ & $\begin{array}{l}0.14 \\
0.04\end{array}$ & $\begin{array}{l}1.35 \\
0.62\end{array}$ \\
\hline $\mathrm{K}\left(\mathrm{mg} \mathrm{L}^{-1}\right)$ & 0.007 & $\begin{array}{l}\text { Lowland } \\
\text { Shield }\end{array}$ & $\begin{array}{l}0.15 \\
0.18\end{array}$ & $\begin{array}{l}0.15 \\
0.17\end{array}$ & $\begin{array}{l}0.25 \\
0.33\end{array}$ & $\begin{array}{l}0.04 \\
0.11\end{array}$ & $\begin{array}{l}0.03 \\
0.05\end{array}$ & $\begin{array}{l}0.22 \\
0.26\end{array}$ \\
\hline $\mathrm{Mg}\left(\mathrm{mg} \mathrm{L}^{-1}\right)$ & 0.028 & $\begin{array}{l}\text { Lowland } \\
\text { Shield }\end{array}$ & $\begin{array}{l}1.21 \\
1.42\end{array}$ & $\begin{array}{l}1.02 \\
1.39\end{array}$ & $\begin{array}{l}5.05 \\
2.32\end{array}$ & $\begin{array}{l}0.19 \\
0.55\end{array}$ & $\begin{array}{l}0.90 \\
0.44\end{array}$ & $\begin{array}{l}0.74 \\
0.31\end{array}$ \\
\hline $\mathrm{SO}_{4}\left(\mathrm{mg} \mathrm{L}^{-1}\right)$ & 0.731 & $\begin{array}{l}\text { Lowland } \\
\text { Shield }\end{array}$ & $\begin{array}{l}0.15 \\
0.23\end{array}$ & $\begin{array}{l}0.08 \\
0.08\end{array}$ & $\begin{array}{l}1.51 \\
2.01\end{array}$ & $\begin{array}{l}0.03 \\
0.03\end{array}$ & $\begin{array}{l}0.21 \\
0.46\end{array}$ & $\begin{array}{l}1.40 \\
2.01\end{array}$ \\
\hline
\end{tabular}


variability, encompassing the variability of the smaller Shield group. However, Lowlands lakes did have significantly $(\mathrm{P}<0.01)$ lower values for some variables $(\mathrm{pH}$, total N, Si and K) than Shield lakes (Tab. 1).

\section{Large scale survey}

The Large Scale Survey lakes showed a much greater range in depths than the ROF lakes (Tabs. 1 and 2). Similar to the ROF lakes, there were large (order of magnitude) ranges between maximum and minimum values in conductivity, colour, inorganic $\mathrm{N}, \mathrm{Ca}, \mathrm{Mg}$, silicate, $\mathrm{K}$, and $\mathrm{SO}_{4}$ (Tab. 2; Supplementary Tab. 3). Lake depth and area were positively correlated $(\mathrm{P}<0.01)$ with $\mathrm{pH}$, conductivity, major cations (Ca, Mg, Na, K, ) silica, and sulphate. Depth was negatively correlated with DOC, colour, total N, TP and Fe (Supplementary Tab. 2b).
Although our focus in this study was on spatial patterns within survey years, we did examine year to year variations in 13 lakes sampled in 2011 and 2012. Chemistry across this lake subset was similar in both years and only 2 variables (TP and FE) exhibited significant $(\mathrm{P}<0.01)$ but slight, pairwise between-year differences (MWU test). Thus, year of sampling was not an important factor affecting our results.

PCA characterized broad-scale patterns and identified the primary sources of chemical variability among the lakes in the Large Scale Survey (Fig. 3). Similar to the ROF lakes, the variables with high positive scores on PC1 (45.1\% of the variation explained) were $\mathrm{pH}(0.329), \mathrm{Mg}$ (0.332), DIC (0.324), conductivity (0.322), and $\mathrm{Ca}$ (0.288). Colour (-0.240), DOC (-0.237) and total N ($0.200)$ scored negatively on $\mathrm{PC} 1$. The variables which scored positively on PC2 (18.2\% of the variation ex-

Tab. 2. Descriptive statistics for lake chemistry and morphometry from the 2012 Large Scale Survey (N=49, 19 Lowlands and 30 Shield).

\begin{tabular}{|c|c|c|c|c|c|c|c|c|}
\hline Variable & $\begin{array}{c}\text { Mann- } \\
\text { Whitney } \\
\text { U-test (P) }\end{array}$ & Region & Mean & Median & Max & Min & $\begin{array}{l}\text { Standard } \\
\text { deviation }\end{array}$ & $\begin{array}{l}\text { Coefficient } \\
\text { of variation }\end{array}$ \\
\hline Lake depth (m) & 0.000 & $\begin{array}{l}\text { Lowland } \\
\text { Shield }\end{array}$ & $\begin{array}{c}3.43 \\
14.17\end{array}$ & $\begin{array}{l}1.90 \\
7.00\end{array}$ & $\begin{array}{l}16.00 \\
70.00\end{array}$ & $\begin{array}{l}1.20 \\
1.80\end{array}$ & $\begin{array}{c}4.09 \\
16.64\end{array}$ & $\begin{array}{l}1.19 \\
1.17\end{array}$ \\
\hline Lake area (ha) & 0.000 & $\begin{array}{c}\text { Lowland } \\
\text { Shield }\end{array}$ & $\begin{array}{l}1047.77 \\
8181.94\end{array}$ & $\begin{array}{c}498.78 \\
2303.62\end{array}$ & $\begin{array}{c}5061.70 \\
62566.00\end{array}$ & $\begin{array}{c}35.78 \\
309.00\end{array}$ & $\begin{array}{c}1231.57 \\
14474.00\end{array}$ & $\begin{array}{l}1.18 \\
1.77\end{array}$ \\
\hline Conductivity $\left(\mu \mathrm{S} \mathrm{cm}^{-1}\right)$ & 0.007 & $\begin{array}{c}\text { Lowland } \\
\text { Shield }\end{array}$ & $\begin{array}{l}61.73 \\
89.65\end{array}$ & $\begin{array}{l}51.00 \\
82.00\end{array}$ & $\begin{array}{l}137.00 \\
232.00\end{array}$ & $\begin{array}{l}21.20 \\
25.40\end{array}$ & $\begin{array}{l}34.95 \\
46.68\end{array}$ & $\begin{array}{l}0.57 \\
0.52\end{array}$ \\
\hline $\mathrm{pH}$ & 0.000 & $\begin{array}{l}\text { Lowland } \\
\text { Shield }\end{array}$ & $\begin{array}{l}7.50 \\
7.84\end{array}$ & $\begin{array}{l}7.43 \\
7.77\end{array}$ & $\begin{array}{l}7.93 \\
8.25\end{array}$ & $\begin{array}{l}6.94 \\
7.17\end{array}$ & - & $\begin{array}{l}- \\
-\end{array}$ \\
\hline $\mathrm{DOC}\left(\mathrm{mg} \mathrm{L}^{-1}\right)$ & 0.000 & $\begin{array}{l}\text { Lowland } \\
\text { Shield }\end{array}$ & $\begin{array}{l}13.50 \\
10.12\end{array}$ & $\begin{array}{l}13.30 \\
11.15\end{array}$ & $\begin{array}{l}18.60 \\
15.40\end{array}$ & $\begin{array}{l}7.80 \\
4.90\end{array}$ & $\begin{array}{l}2.90 \\
2.79\end{array}$ & $\begin{array}{l}0.21 \\
0.28\end{array}$ \\
\hline True colour (TCU) & 0.000 & $\begin{array}{l}\text { Lowland } \\
\text { Shield }\end{array}$ & $\begin{array}{l}86.02 \\
43.51\end{array}$ & $\begin{array}{l}83.00 \\
41.10\end{array}$ & $\begin{array}{l}155.00 \\
127.00\end{array}$ & $\begin{array}{c}31.00 \\
5.20\end{array}$ & $\begin{array}{l}34.16 \\
28.29\end{array}$ & $\begin{array}{l}0.40 \\
0.65\end{array}$ \\
\hline Inorganic $\mathrm{N}\left(\mu \mathrm{g} \mathrm{L}^{-1}\right)$ & 0.194 & $\begin{array}{c}\text { Lowland } \\
\text { Shield }\end{array}$ & $\begin{array}{l}15.36 \\
12.54\end{array}$ & $\begin{array}{c}15.60 \\
9.95\end{array}$ & $\begin{array}{l}25.20 \\
53.40\end{array}$ & $\begin{array}{l}8.00 \\
3.60\end{array}$ & $\begin{array}{c}5.32 \\
10.05\end{array}$ & $\begin{array}{l}0.35 \\
0.80\end{array}$ \\
\hline Total N $\left(\mu \mathrm{g} \mathrm{L}^{-1}\right)$ & 0.029 & $\begin{array}{c}\text { Lowland } \\
\text { Shield }\end{array}$ & $\begin{array}{l}391.05 \\
350.50\end{array}$ & $\begin{array}{l}384.00 \\
355.50\end{array}$ & $\begin{array}{l}513.00 \\
540.00\end{array}$ & $\begin{array}{l}297.00 \\
163.00\end{array}$ & $\begin{array}{l}63.48 \\
77.51\end{array}$ & $\begin{array}{l}0.16 \\
0.22\end{array}$ \\
\hline Total $\mathrm{P}\left(\mu \mathrm{g} \mathrm{L}^{-1}\right)$ & 0.005 & $\begin{array}{l}\text { Lowland } \\
\text { Shield }\end{array}$ & $\begin{array}{l}15.36 \\
12.54\end{array}$ & $\begin{array}{c}15.60 \\
9.95\end{array}$ & $\begin{array}{l}25.20 \\
53.40\end{array}$ & $\begin{array}{l}8.00 \\
3.60\end{array}$ & $\begin{array}{c}5.32 \\
10.05\end{array}$ & $\begin{array}{l}0.35 \\
0.80\end{array}$ \\
\hline Reactive Si (mg L $\left.{ }^{-1}\right)$ & 0.000 & $\begin{array}{c}\text { Lowland } \\
\text { Shield }\end{array}$ & $\begin{array}{l}0.33 \\
1.02 \\
\end{array}$ & $\begin{array}{l}0.26 \\
0.92\end{array}$ & $\begin{array}{l}1.46 \\
2.00\end{array}$ & $\begin{array}{l}0.02 \\
0.02\end{array}$ & $\begin{array}{l}0.34 \\
0.57\end{array}$ & $\begin{array}{l}1.03 \\
0.56\end{array}$ \\
\hline $\mathrm{Ca}\left(\mathrm{mg} \mathrm{L}^{-1}\right)$ & 0.024 & $\begin{array}{c}\text { Lowland } \\
\text { Shield }\end{array}$ & $\begin{array}{l}10.14 \\
12.92\end{array}$ & $\begin{array}{c}7.80 \\
12.25\end{array}$ & $\begin{array}{l}28.30 \\
34.90\end{array}$ & $\begin{array}{l}3.14 \\
2.16\end{array}$ & $\begin{array}{l}6.78 \\
7.47 \\
\end{array}$ & $\begin{array}{l}0.67 \\
0.58\end{array}$ \\
\hline $\mathrm{Cl}\left(\mathrm{mg} \mathrm{L}^{-1}\right)$ & 0.505 & $\begin{array}{l}\text { Lowland } \\
\text { Shield }\end{array}$ & $\begin{array}{l}0.56 \\
0.30\end{array}$ & $\begin{array}{l}0.22 \\
0.24\end{array}$ & $\begin{array}{l}2.45 \\
1.11\end{array}$ & $\begin{array}{l}0.11 \\
0.10\end{array}$ & $\begin{array}{l}0.72 \\
0.23\end{array}$ & $\begin{array}{l}1.29 \\
0.77\end{array}$ \\
\hline $\mathrm{Fe}\left(\mathrm{mg} \mathrm{L}^{-1}\right)$ & 0.000 & $\begin{array}{l}\text { Lowland } \\
\text { Shield }\end{array}$ & $\begin{array}{l}0.20 \\
0.15\end{array}$ & $\begin{array}{l}0.14 \\
0.08\end{array}$ & $\begin{array}{l}0.51 \\
1.43\end{array}$ & $\begin{array}{l}0.06 \\
0.01\end{array}$ & $\begin{array}{l}0.15 \\
0.28\end{array}$ & $\begin{array}{l}0.75 \\
1.87\end{array}$ \\
\hline $\mathrm{K}\left(\mathrm{mg} \mathrm{L}^{-1}\right)$ & 0.000 & $\begin{array}{c}\text { Lowland } \\
\text { Shield }\end{array}$ & $\begin{array}{l}0.16 \\
0.49\end{array}$ & $\begin{array}{l}0.15 \\
0.43\end{array}$ & $\begin{array}{l}0.26 \\
1.04\end{array}$ & $\begin{array}{l}0.09 \\
0.18\end{array}$ & $\begin{array}{l}0.04 \\
0.21\end{array}$ & $\begin{array}{l}0.25 \\
0.43\end{array}$ \\
\hline $\mathrm{Mg}\left(\mathrm{mg} \mathrm{L}^{-1}\right)$ & 0.000 & $\begin{array}{c}\text { Lowland } \\
\text { Shield }\end{array}$ & $\begin{array}{l}1.44 \\
2.79\end{array}$ & $\begin{array}{l}1.27 \\
2.61\end{array}$ & $\begin{array}{l}2.81 \\
7.92\end{array}$ & $\begin{array}{l}0.48 \\
0.93\end{array}$ & $\begin{array}{l}0.74 \\
1.59\end{array}$ & $\begin{array}{l}0.51 \\
0.57\end{array}$ \\
\hline $\mathrm{SO}_{4}\left(\mathrm{mg} \mathrm{L}^{-1}\right)$ & 0.000 & $\begin{array}{l}\text { Lowland } \\
\text { Shield }\end{array}$ & $\begin{array}{l}0.17 \\
0.65\end{array}$ & $\begin{array}{l}0.15 \\
0.53\end{array}$ & $\begin{array}{l}0.35 \\
1.90\end{array}$ & $\begin{array}{l}0.05 \\
0.10\end{array}$ & $\begin{array}{l}0.08 \\
0.48\end{array}$ & $\begin{array}{l}0.47 \\
0.73\end{array}$ \\
\hline
\end{tabular}


plained) were $\mathrm{Fe}(0.373)$, DOC (0.313), TP (0.293), total $\mathrm{N}(0.286)$ and colour $(0.248) . \mathrm{SO}_{4}(-0.356)$ scored negatively on PC2. There was clear separation in ordination space between Shield and Lowlands lakes (Fig. 3). ANOSIM also indicated a clear overall difference (global $\mathrm{R}=0.375, \mathrm{P}<0.001$ ) between the lake groups. Lowlands lakes had shallower depths, smaller areas, lower $\mathrm{pH}$, conductivity and major ions $\left(\mathrm{Ca}, \mathrm{K}, \mathrm{Mg}, \mathrm{SO}_{4}\right)$ and higher DOC, colour and TP $(\mathrm{P}<0.01)$ than Shield lakes (Tab. 2).

Considering only Shield lakes, lakes from our 2012 Large Scale Survey had generally higher $\mathrm{pH}$, ionic strength $(\mathrm{Ca}, \mathrm{Mg})$ and TP concentrations than northwestern Ontario Shield lakes further south (Keller and Pitblado, 1989; Tab. 3).

\section{DISCUSSION}

\section{Variability in ROF area chemistry}

The lakes of the ROF region displayed high variability considering that the 2011 survey only covered an area of $100 \times 45 \mathrm{~km}$ (Fig. 1). In contrast, the 2012 Large Scale Survey covered an area 72 times larger $(740 \mathrm{~km}$ x $420 \mathrm{~km})$. The lakes in the ROF Survey were all shallow ( $<5 \mathrm{~m}$ deep) but ranged in surface area from $\sim 5$ ha to over 1000 ha (Tab. 1). These lakes have proportionately larger littoral habitats and may be more productive than deeper lakes due to the greater percentage of euphotic zone. Many chemical variables $\left(\mathrm{pH}\right.$, conductivity, total $\mathrm{N}, \mathrm{Si}, \mathrm{Ca}, \mathrm{Mg}, \mathrm{SO}_{4}$ ) actually varied more within the ROF area than across the Large Scale Survey (Tabs. 1 and 2). Chemistry variation in both surveys was primarily driven by ionic strength $(\mathrm{pH}$, conductivity, $\mathrm{Ca}$ and $\mathrm{Mg}$ ) and secondly by organic content (colour, DOC). Similar PCA derived water chemistry gradients have been reported for other northern lake studies (Swadling et al., 2001; Medeiros et al., 2012)

To put this variability in context, the ROF lakes span the majority of the $\mathrm{pH}$ range obtained from nearly 6000 Ontario lakes surveyed in the 1980s (Neary et al., 1990) $(\mathrm{N}=5982, \mathrm{pH}=3.0-9.8)$. Compared with other parameters from Neary et al., (1990), including DOC $(0.1-58 \mathrm{mg} \mathrm{L}$ $\left.{ }^{1}, \mathrm{~N}=2581\right), \mathrm{Ca}\left(0.1-70.6 \mathrm{mg} \mathrm{L}^{-1}, \mathrm{~N}=3702\right), \mathrm{Mg}(0.5-23.7$ $\left.\mathrm{mg} \mathrm{L}{ }^{-1}, \mathrm{~N}=3591\right), \mathrm{K}\left(0.04-2.98 \mathrm{mg} \mathrm{L}^{-1}, \mathrm{~N}=3153\right)$ and $\mathrm{SO}_{4}$ (0.3-34.5 $\mathrm{mg} \mathrm{L}^{-1}, \mathrm{~N}=3599$ ), the 98 ROF lakes (Tab. 1) covered roughly half of the range obtained for DOC and $\mathrm{Ca}$, and a smaller $(<20 \%)$ portion of the ranges of $\mathrm{Mg}, \mathrm{K}$, and $\mathrm{SO}_{4}$. Nitrogen levels (especially inorganic $\mathrm{N}$ ) were generally much lower than those reported in lakes from Neary et al. (1990).

Considering the large degree of variation in lake chemistry within the ROF area (Tab. 1), and given that the changes in the overall geological characteristics asso-

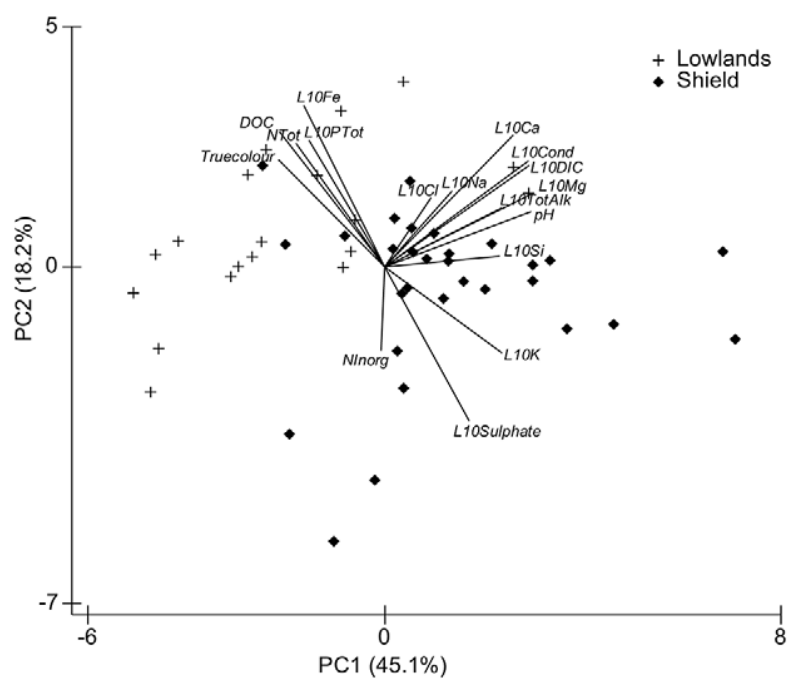

Fig. 3. PCA of 2012 water chemistry and morphometry for the Large Scale Survey lakes (N=49, 30 Shield and 19 Lowlands).

Tab. 3. Comparison of chemistry variables for Shield lakes south of $50^{\circ} \mathrm{N}$ latitude sampled in 1981 from Keller and Pitblado (1989) and the Shield lakes sampled in the 2012 Large Scale Survey (1981, N=137; 2012, N=30). All variables were significantly different based on Mann Whitney U tests for differences between means $(\mathrm{P}<0.01)$.

\begin{tabular}{lcc} 
& Keller and Pitblado (1989) & Large Scale Survey (2012) \\
$\mathrm{pH}$ & $6.84(6.07-7.75)$ & $7.84(7.17-8.25)$ \\
\hline Conductivity $\left(\mu \mathrm{S} \mathrm{cm}^{-1}\right)$ & $33(21-116)$ & $90(25-232)$ \\
\hline $\mathrm{Ca}\left(\mathrm{mg} \mathrm{L}^{-1}\right)$ & $3.6(1.4-14.3)$ & $12.9(2.2-34.9)$ \\
\hline $\mathrm{Mg}\left(\mathrm{mg} \mathrm{L}^{-1}\right)$ & $1.2(0.8-4.3)$ & $2.8(1.0-7.9)$ \\
\hline $\mathrm{Cl}\left(\mathrm{mg} \mathrm{L}^{-1}\right)$ & $0.5(0.20-4.4)$ & $0.41(0.10-2.45)$ \\
\hline $\mathrm{Na}\left(\mathrm{mg} \mathrm{L}^{-1}\right)$ & $1.0(0.6-2.8)$ & $0.7(0.4-1.5)$ \\
\hline $\mathrm{K}\left(\mathrm{mg} \mathrm{L}^{-1}\right)$ & $1.5(0.2-4.4)$ & $0.5(0.2-1.0)$ \\
\hline $\mathrm{SO}_{4}\left(\mathrm{mg} \mathrm{L}^{-1}\right)$ & $3.7(1.9-6.7)$ & $0.7(0.1-1.9)$ \\
\hline Total P $\left(\mu \mathrm{g} \mathrm{L}^{-1}\right)$ & $9(2-36)$ & $13(4-53)$ \\
\hline
\end{tabular}


ciated with the different physiographic regions are not reflected in changes in water chemistry, lake chemistry appears to be decoupled to varying degrees from reactions with bedrock and overlying till by the extensive peat deposits in this area (Lacelle, 1997; Tarnocai 1997). However, this isolation will vary as localized surficial deposits interact with groundwater where the peat layer is thinner. The effects of peat cover are greatly complicated by the patchwork of bogs and fens present throughout this landscape (Riley, 2011; Barnett et al., 2013). Fens, which have groundwater connectivity, can transport elements from subsurface till into lakes. Bogs are isolated from groundwater inputs, and therefore are disconnected from underlying lithological influences but may provide considerable plant-derived organic carbon to lakes. Differences in the proportions of different wetland types in individual lake watersheds will affect solute concentrations, acidity and organic matter, ultimately contributing to chemically diverse lakes. Such watershed effects may, however, be complex given the large variability in the vegetational (Riley, 2011) and hydrological (Orlova and Branfireun, 2014; Turner et al., 2014; White et al. 2014) characteristics of Lowlands ecosystems. Hydrological studies in the Yukon (Turner et al., 2014) have indicated that snowmelt dominated lakes typically have catchments characterized by woodlands and tall shrubs, while catchments characterized by dwarf shrubs and sparse vegetation are more susceptible to evaporative effects, especially in dry years.

Studies in other peatland regions have demonstrated clear associations between the extent of wetlands within watersheds and organic carbon concentrations in lakes (Kortelainen, 1993), and have documented increasing lake/pond acidity with increasing DOC/colour (Halsey et al., 1997) as was observed in the ROF lakes. This suggests a strong role for organic acids in determining surface water acidity in the ROF area. While bogs typically have high acidity, it has been suggested that less acidic fens may ultimately have greater downstream effects because of greater water throughflow (Halsey et al., 1997). Similar to lakes in other northern landscapes with thick peat cover, some lakes in the ROF area were moderately acidic $(\mathrm{pH}$ $<6,10 \%)$ and a few were highly acidic $(\mathrm{pH}<5,5 \%)$. In comparison, in a survey of 29 lakes in northern Alberta peatlands, only $2(7 \%)$ had $\mathrm{pH}<5$, and these were the only lakes with $\mathrm{pH}<6$ (Halsey et al., 1997). In a survey of 37 lakes and ponds in Subarctic Quebec, 9 (24\%) and 3 (8\%) waterbodies were below $\mathrm{pH} 6$, and 5, respectively (Swadling et al. 2001). Overall, these results suggest that in thick northern peatlands such as the ROF area, fens, rather than the more acidic bogs have a dominant influence on lake water acidity, in agreement with the findings of Halsey et al. (1997). Most (90\%) of the ROF lakes had $\mathrm{pH}>6$, typical of rich fens, not the more acidic bogs and poor fens, with $37 \%$ of the lakes in the 6 to $7 \mathrm{pH}$ range.
In contrast, lakes and ponds further north in arctic and subarctic environments, with less peat covering the till and bedrock, are usually near-neutral or alkaline (Rautio et al. 2011; Medeiros et al., 2012). For example, 32 ponds in northern Manitoba along the Hudson Bay coast $\sim 800$ $\mathrm{km}$ northwest of the ROF all had $\mathrm{pH}>7$ (Bos and Pellat, 2012). These ponds, in an area with generally lower peat thickness than the ROF area (Tarnocai, 1997) also had, on average, higher $\mathrm{Ca}$ concentrations $\left(26.0 \mathrm{mg} \mathrm{L}^{-1}\right)$ than ROF lakes $\left(11.8 \mathrm{mg} \mathrm{L}^{-1}\right)$ indicating a generally greater influence by underlying calcareous till and bedrock on waters further north.

In permafrost regions thermokarst processes can greatly affect the chemistry of lakes and ponds (Bouchard et al., 2014; Manasypov et al., 2014; Coleman et al., 2015). Unfortunately, we have no information to assess the actual extent of thermokarst effects on ROF lakes. However, it is very likely that permafrost does influence the variability in lake chemistry within the ROF area. The ROF study lakes were located across a zone where permafrost extent varies considerably, from no permafrost to a few $(<10 \%)$ isolated patches, becoming more prevalent north and eastward to a moderate (as much as $50 \%$ ), but sporadic discontinuous distribution (Heginbottom et al., 1995), which increases in the direction of the climatic influence of Hudson Bay. The extensive peat overburden in this region (Tarnocai, 1997), acts as a conduction pathway for groundwater (Devito et al., 1996). When combined with a range of differing permafrost densities throughout this region (Heginbottom et al., 1995), a variable permeable/impermeable barrier is created. This may isolate flow between water bodies, much like water bodies on a floodplain system with temporary linkages. Thus, permafrost may allow lakes to diverge chemically as the flow of nutrients and organic matter is restricted by stagnant hydrologic conditions that isolate the lakes, and then change again when connections are re-established through rainfall events that promote subsurface flow (Stieglitz et al., 2003). This effect may be further accentuated by thawing permafrost as a result of warming from climatic change (Anisimov and Nelson, 1996; Osterkamp and Romanovsky, 1999), which would open up new hydrological connections. The ever-changing nature of the hydrologic landscape in permafrost areas promotes divergence (Stieglitz et al., 2003), which may, in part, account for the high variability we observed in lake chemistry.

\section{Large-scale differences in lakes chemistry}

In contrast to lakes in the ROF transitional area, the lakes in the Large Scale Survey did show a clear separation between Shield and Lowlands lakes (Fig. 3; ANOSIM $\mathrm{P}<0.001$ ). Shield lakes are typically deep, cold and clear. The Shield lakes from the Large Scale Survey exhibited these general characteristics in that they had 
lower DOC, colour and TP, which suggests that they are less productive than the 2012 Lowlands lakes. They were deeper than Lowlands lakes, which will directly affect the relative influences of external forces such as wind and solar radiation, in turn affecting thermal characteristics and lake chemistry.

The distinct separation of Shield and Lowlands lakes in the Large Scale Survey is related to the scale of study. While the ROF lakes are located in a complicated mosaic of varying wetland and permafrost conditions within a relatively restricted area, the Large Scale Survey lakes cover a wide gradient in such watershed characteristics over a span of hundreds of kilometers. The extent of peatland cover differed to a large degree between Shield and Lowlands lakes in the Large Scale Survey. Peatlands were less extensive across the Shield than in the Lowlands (Tarnocai, 1997). Thus, the potential influence of organic acid inputs and isolation as a result of a thick peat layer, which varied considerably throughout the ROF survey area, was much reduced for Shield lakes located further west. While permafrost likely affected the more northerly Lowlands lakes, it was absent from the catchments of Shield lakes further south.

Northern Shield lakes $\left(>50^{\circ} \mathrm{N}\right)$ are of a different character than other Shield lakes. The Shield lakes from the Large Scale Survey showed higher $\mathrm{pH}$, ionic strength, $\mathrm{Ca}$, $\mathrm{Mg}$ and TP than previously sampled northwestern Ontario Shield lakes (Tab. 3). The explanation for these differences is the presence of extensive calcareous end moraine and lacustrine deposits left during the end of the last ice age, which are more prevalent on the Canadian Shield in northwestern Ontario above $50^{\circ} \mathrm{N}$ (Royal Commission on the Northern Environment, 1985). Newer maps also confirm this pattern (Four Rivers Matawa Environmental Services Group, 2013). Expectations for Shield lake chemistry in Ontario must be broadened to include higher $\mathrm{pH}$, ionic strength, and TP concentrations, as well as high variability in Shield lakes north of $50^{\circ} \mathrm{N}$.

\section{CONCLUSIONS}

Within the ROF area, the chemical distinction between Shield lakes and Lowlands lakes was not clear; however, across a much broader geographical area, the chemistry differences between lakes in these two physiographic regions were apparent. In the transitional ROF area, lake chemistry is largely decoupled from influences from bedrock and surficial deposits by an extensive, but highly variable peat layer. This leads to variable influences from the underlying glacial till and marine deposits that are affected by the differing nature of the wetland drainage in this very heterogeneous peatland landscape of bogs and fens. Large variations in the extent of permafrost within this area are a further complicating factor, likely contribut- ing to the high variability in lake chemistry. At the broader scale of the Large Scale Survey the clear Shield/Lowlands differences reflected broad spatial changes in geology and large gradients in the extent of peatlands and permafrost across the north.

Importantly, our study identified differences between the chemical properties of Shield lakes north of $50^{\circ} \mathrm{N}$ and Shield lakes further south in Ontario, which expands the current understanding of Shield lake chemistry in a fundamental way. The existing perception of Shield lakes in Ontario must be broadened to include lakes which are comparatively high in $\mathrm{Ca}, \mathrm{Mg}, \mathrm{TP}, \mathrm{pH}$, and conductivity. Some Shield lakes near the Shield/Lowlands transition are uncharacteristically shallow and highly coloured.

We have only sampled a very small fraction of the many thousands of lakes in northern Ontario, and for interpretation of patterns we have relied on the available coarse-scale landscape mapping. Much additional lake and watershed sampling needs to be done to increase our knowledge base for northern aquatic ecosystems. Detailed, process-oriented studies examining landscape/ chemistry linkages for specific lakes and their watersheds are needed to better understand the mechanisms controlling northern lake chemistry. Given the shallow, very dynamic nature of many of these lakes, assessments of temporal chemistry variability are needed. The vast, remote nature of northern Ontario creates major logistical difficulties for conventional surveys. To augment field surveys, and increase spatial and temporal data collection opportunities in a cost effective manner, the use of remote sensing and remote monitoring techniques for assessments of these northern systems needs to be explored. It is hoped that the results presented here will stimulate further research on northern lakes, help advance the scientific understanding of northern aquatic ecosystems, and aid in the development of comprehensive future assessment and monitoring programs.

\section{ACKNOWLEDGMENTS}

These studies were completed with the support of: the Ontario Ministry of the Environment and Climate Change through the Climate Change and Multiple Stressor Aquatic Research Program at Laurentian University; the Ontario Geological Survey; the Wildlife Conservation Society through the Garfield Weston Foundation; and NSERC through the Canadian Network for Aquatic Ecosystem Services. Adam Jeziorski, Kathryn Hargan, and Chantal Sarrazin-Delay provided valuable assistance in the field and Xavier Sagutch helped with survey planning. We thank Kim Armstrong and Jeff Amos (MNRF) for access to the BSM data. We are grateful for all the assistance provided by the Eabametoong First Nation at Fort Hope during the 2012 survey. 


\section{REFERENCES}

Anderson MJ, 2001. A new method for non-parametric multivariate analysis of variance. Austral Ecology 26:32-46.

Anisimov OA, Nelson FE, 1996. Permafrost distribution in the northern hemisphere under scenarios of climatic change. Global Planet. Change 14:9-72.

Barnett PJ, Yeung KH, McCallum JD, 2013. Surficial geology of the Lansdowne House Area Northeast, Northern Ontario. Ontario Geological Survey, preliminary Map P3697, scale 1:100 000 .

Bos DG, Pellatt MG, 2012. The water chemistry of shallow ponds around Wapusk National Park of Canada, Hudson Bay Lowlands. Can. Water Res. J. 37:163-175.

Bouchard F, Francus P, Pienitz R, Laurion I, Feyte S, 2014. Subarctic thermokarst ponds: investigating recent landscape evolution and sediment dynamics in thawed permafrost of northern Quebec (Canada). Arct. Antarct. Alpi. Res. 46:251-271.

Chapman MG, Underwood AJ, 1999. Ecological patterns in multivariate assemblages: information and interpretation of negative values in ANOSIM tests. Mar. Ecol. Proger. S. 180:257-265.

Clarke KR, 1993. Non-parametric multivariate analyses of changes in community structure. Austral. J. Ecol. 18:117-143.

Coleman KA, Palmer MJ, Korosi JB, Kokelj SV, Jackson K, Hargan K.E, Mustaphi CJ, Thienpont JR, Kimpe LE, Blais JM, Pisaric MFJ, Smol JP, 2015. Tracking the impacts of recent warming and thaw of permafrost peatlands on aquatic ecosystems: a multi-proxy approach using remote sensing and lake sediments. Boreal Environ. Res. 20:363-377.

Colombo SJ, McKenney DW, Lawrence KM, Gray PA, 2007. Climate Change Projections for Ontario: Practical Information for Policymakers and Planners. Climate Change Research Report CCRR-05. Applied Research and Development Branch - Ontario Ministry of Natural Resources, Sault Ste. Marie, Ontario: 37 pp.

Devito K, Hill AR, Roulet N, 1996. Groundwater-surface water interactions in headwater forested wetlands of the Canadian Shield. J. Hydrol. 181:127-147.

Dyer RD, 2011. Project Unit 11-024. McFaulds Lake Area Lake Sediment and Water Pilot Study, Northern Ontario. In, Summary of Field Work and Other Activities 2011, Open File Report 6270, p. 25.1-25.6. Ontario Geological Survey, Sudbury, Ontario.

Dyer RD, Handley LA, 2013. McFaulds Lake ("Ring of Fire") Area High Density Lake Sediment and Water Survey, Far North, Ontario. Ontario Geological Survey, Sudbury, Ontario.

Far North Science Advisory Panel, 2010. Science for a Changing Far North, the Report of the Far North Science Advisory Panel. A report submitted to the Ontario Ministry of Natural Resources: 142 p.

Four Rivers Matawa Environmental Services Group, 2013. Surficial Geology and Infrastructure (1:700,000 scale map) NAD1983 UTM Zone 16N. Ontario Ministry of Natural Resources, Ontario Ministry of Northern Development and Mines.

Halsey LA, Vitt DH, Trew DO, 1997. Influence of peatlands on the acidity of lakes in northeastern Alberta, Canada. Water Air Soil Poll. 96:17-38.

Heginbottom JA, Dubreuil MA, Harker PA, 1995. Canada Per- mafrost. Geomatics Canada. National Atlas Information Service and Geological Survey of Canada, Ottawa.

Hjartarson J, McGuinty L, Boutileir S, Majernikova E, 2014. Beneath the surface: uncovering the economic potential of Ontario's Ring of Fire. Ontario Chamber of Commerce Report. ISBN 978-1-928052-01-2.

Jeziorski A, Keller B, Dyer RD, Paterson AM, Smol JP, 2015. Differences among modern-day and historical cladoceran communities from the "Ring of Fire" lake region of northern Ontario: Identifying responses to climate warming. Fund. Appl. Limnol. 186:203-216.

Keller W, Pitblado JR, 1989. The distribution of crustacean zooplankton in northern Ontario, Canada. J. Biogeogr. 16: 249-259.

Keller W, Conlon M, 1994. Crustacean zooplankton communities and lake morphometry in Precambrian Shield lakes. Can. J. Fish. Aquat. Sci. 51:2424-2434.

Kortelainen P, 1993. Content of total organic carbon in Finnish lakes and its relationship to catchment characteristics. Can. J. Fish. Aquat. Sci. 50:1477-1483.

Lacelle B, 1997. Canada's soil organic carbon database, p. 83101. In: R. Lal, J.M. Kimble, R.F. Follett and B.A. Stewart (eds.), Soil processes and the carbon cycle (Advances in soil science). CRC Press, New York.

Manasypov RM, Pokrovsky OS, Kirpotin SN, Shirokova LS, 2014. Thermokarst lake waters across the permafrost zones of western Siberia. Cryosphere 8:1177-1193.

Medeiros AS, Biastoch RG, Luszczek CE, Wang XA, Muir DCG, Quinlan R., 2012. Patterns in the limnology of lakes and ponds across multiple local and regional environmental gradients in the eastern Canadian arctic. Inland Waters 2:59-76.

Metsaranta RT, Houlé MG, 2012. Progress on the McFaulds Lake ("Ring of Fire") Regional Compilation and Bedrock Mapping Project, p. 43-1 to 43-9. In: Summary of Field Work and Other Activities 2012, Ontario Geological Survey Open File Report 6280.

Moran M, 2003. Arguments for rejecting the sequential Bonferroni in ecological studies. Oikos 100:403-405.

Neary BP, Dillon PJ, Munro JR, Clark BJ, 1990. The acidification of Ontario Lakes: an assessment of their sensitivity and current status with respect to biological damage. Ontario Ministry of the Environment Tech. Report, Toronto, Ontario: $171 \mathrm{pp}$.

OGS (Ontario Geological Survey), 2007. Major and Trace Element Analysis of Water Samples by Inductively Coupled Plasma Atomic Emission Spectrometry (ICP-AES) at the Geosciences Laboratories: Preliminary Data. In, Ontario Geological Survey, Open File Report 6213, ISBN 978-14249-5410-0.

Oliver I, Beattie AJ, 1996. Invertebrate morphospecies as surrogates for species: a case study. Conserv. Biol. 10: 99-09.

OMOE (Ontario Ministry of the Environment), 1983. Handbook of Analytical Methods for Environmental Samples. Laboratory Services Branch, Ontario Ministry of Environment and Energy. Volumes 1 and 2. Queen's Printer for Ontario, Toronto, Ontario.

Orlova J, Branfireun BA, 2014. Surface water and groundwater contributions to streamflow in the James Bay Lowland, Canada. Arct. Antarct. Alp. Res. 46:236-250.

Osterkamp TE, Romanovsky VE, 1999. Evidence for warming 
and thawing of discontinuous permafrost in Alaska. Permafrost Periglac. 10:17-37.

Paterson AM, Keller W (Bill), Rühland KM, Jones FC, Winter JG, 2014. An exploratory survey of summer water chemistry and plankton communities in lakes near the Sutton River, Hudson Bay Lowlands, Ontario, Canada. Arct. Antarct. Alp. Res 46:121-138.

Rautio M, Dufresne F, Laurion I, Bonilla S, Vincent WF, Christofferson KS, 2011. Shallow freshwater ecosystems of the circumpolar Arctic. Ecoscience 18:204-222.

Riley JL, 2011. Wetlands of the Hudson Bay Lowland: a regional overview. Nature Conservancy of Canada, Toronto, Ontario: $156 \mathrm{p}$.

Royal Commission on the Northern Environment, 1985. North of 50: an Atlas of Far Northern Ontario. University of Toronto Press, Toronto, Ontario: $119 \mathrm{p}$.

Rühland KM, Paterson AM, Keller W, Michelutti N, Smol JP, 2013. Global warming triggers the loss of a key Arctic refugium. P. Roy. Soc. B-Biol. 280:20131887.

Rühland K, Hargan K, Jeziorski A, Paterson AM, Keller W, Smol JP, 2014. A multi-trophic exploratory survey of recent environmental change using lake sediments in the Hudson Bay Lowlands, Ontario. Arct. Antarct. Alp. Res 46:139-158.

Ryder RA, 1964. Chemical characteristics of Ontario lakes as related to glacial history. T. Am. Fish. Soc. 93:260-268.

Sandstrom S, Rawson M, Lester N, 2011. Manual of Instructions for Broad- scale Fish Community Monitoring using North
American Gillnets (NA1) and Ontario Small Mesh Gillnets (ON2) Ver. 2011.1. Ontario Ministry of Natural Resources, Peterborough, Ontario: 35 pp.

Stieglitz M, Shaman J, McNamara J, Engel V, Shanley J, Kling $\mathrm{GW}, 2003$. An approach to understanding hydrologic connectivity on the hillslope and the implications for nutrient transport. Global Biogeochem. Cycles 17:1105.

Swadling KM, Gibson JAE, Pienitz R, Vincent WF, 2001. Biogeography of copepods in lakes and ponds of subarctic Quebec, Canada. Hydrobiologia 453/454:341-350.

Tarnocai C, 1997. The amount of organic carbon in various soil orders and ecological provinces in Canada, p. 81-82. In: R. Lal, J.M. Kimble, R.F. Follett and B.A. Stewart (eds.), Soil processes and the carbon cycle (Advances in soil science). CRC Press, New York.

Turner KW, Wolfe BB, Edwards TWD, Lantz TC, Hall R.I, Larocque G, 2014. Controls on water balance in shallow thermokarst lakes and their relations with catchment characteristics: a multi-year, landscape-scale assessment based on water isotope tracers and remote sensing in Old Crow Flats, Yukon (Canada). Glob. Change Biol. 20:1585-1603.

White J, Hall RI, Wolfe BB, Light EM, Macrae ML, Fishback L, 2014. Hydrological connectivity influences seasonal patterns of limnological conditions in shallow tundra ponds of the western Hudson Bay Lowlands. Arct. Antarct. Alp. Res. 46:218-235. 